Imagem corporal, estado nutricional e comportamento com relação ao peso entre adolescentes brasileiros

\author{
Body image, nutritional status and practices for weight control \\ among Brazilian adolescents
}

\author{
Inês Rugani Ribeiro de Castro ${ }^{1}$ \\ Renata Bertazzi Levy ${ }^{2}$ \\ Letícia de Oliveira Cardoso ${ }^{3}$ \\ Michelle Delboni dos Passos ${ }^{1}$ \\ Luciana M onteiro Vasconcelos Sardinha ${ }^{4}$ \\ Letícia FerreiraTavares $^{1}$ \\ Sheila Potente Dutra ${ }^{5}$ \\ André $M$ artins ${ }^{6}$
}

${ }^{1}$ Departamento de N utrição Social, Universidade do Estado do Rio deJaneiro. Rua São Francisco Xavier 524, Pavilhão João Lyra Filho, 120 andar, bloco D, sala 12023. 20559-900

Rio deJaneiro RJ. inesrrc@uol.com.br

${ }^{2}$ Departamento deM edicina Preventiva, Faculdade de

M edicina, U niversidade de São Paulo.

${ }^{3}$ Departamento de

Epidemiologia e M étodos Quantitativos em Saúde,

Escola Nacional de Saúde Pública Sergio Arouca,

Fundação O swaldo Cruz.

${ }^{4}$ Secretaria de Vigilância em Saúde, M inistério da

Saúde.

${ }^{5}$ Departamento de N utrição Social, Faculdadede Nutrição, Universidade Federal Fluminense. ${ }^{6}$ Instituto Brasileiro de Geografia e Estatística.
Abstract The objective of this work is to describe the agreement between body image (BI) and nutritional status (NS) as well as to verify the association of $\mathrm{BI}$ and NS with behaviors regarding body weight (BRBW) among Brazilian adolescents. In 2009, a random sample of students in 9th year at public and private schools from 26 Brazilian state capitals and Federal District was studied. It was used a self-administered questionnaire with information about BI, BRBW and socioeconomic factors. Weight and height were measured. Logistic regression analyses were performed to evaluate associations between variables and weighted kappa test was used to verify the agreement between NS and BI. Twenty-four percent of the adolescents had excessive weight (EW) and $17.7 \%$ considered themselves fat. Almost 2/3 performed some action to weight control and $7 \%$ showed extreme behavior for weight control (EBWC). Low agreement between the NS and BI was observed. Nearly half and $27 \%$ of those with EW considered themselves as having normal weight and being thin, respectively. Greater proportion of EW adolescents had EBWC when compared with those with normal weight and underweight. The same was observed in relation to BI. Low agreement between EN and BI was observed. Practices for weight control were observed even among individuals with adequate NS.

Key words Body image, Nutritional status, Weight control, Adolescents
Resumo 0 objetivo deste artigo é descrever a concordância entre imagem corporal (IC) e estado nutricional (EN) e verificar a associação de IC edeEN com comportamentos relacionados ao peso corporal (CRPC) entre adolescentes brasileiros. Estudou-se, em 2009, amostra representativa de alunos do 9o ano do ensino fundamental de escolas públicas eprivadas das 26 capitais brasileirase do Distrito Federal. Utilizou-se questionário autoaplicável sobre IC, CRPC e dados sociodemográficos eforam aferidos peso ealtura. U tilizou-se o testeKappa ponderado (KP) para exameda concordância entre EN e IC. Análises de regressão logística foram realizadas para exame da associação entre as variáveis. Do total de adolescentes, $24 \%$ apresentavam excesso depeso (EP), quase $2 /$ 3 deles tinham alguma atitude para controlar 0 peso e $7 \%$ realizavam práticas extremas para redução do peso (PECP). Foi baixa a concordância entre EN eIC $(K P=0,33)$. Q uase metade dos alunos com EP considerava-se com peso adequado e $27 \%$, magros. M aior proporção de adolescentes com EP fazia uso de PECP, quando comparados àqueles de peso adequado e baixo peso. 0 mesmo foi observado em relação à IC. Conclusão: foi baixa a concordância entreEN eIC. Atitudes de controle de peso ePECP foram frequentes mesmo entre indivíduos com EN adequado.

Palavras-chave Imagem corporal, Estado nutricional, Controle de peso, Adolescentes 
Introdução

Imagem corporal pode ser definida como a percepção que o sujeito tem do próprio corpo com base nas sensações e experiências vividas ao longo da vida'. Ela pode ser influenciada por inúmeros fatores de origem física, psicológica, ambiental e cultural no âmbito da subjetividade de cada ser humano, tais como sexo, idade, meios de comunicação, crenças, raça e valores ${ }^{1}$. É uma espécie de "fotografia mental" que a pessoa elabora sobre a sua aparência física ${ }^{2}$ e pode ser construída ou destruída por tentativas que buscam uma imagem e um corpo ideais ${ }^{3}$.

Embora o concei to de beleza e o padrão ideal de corpo tenham se modificado ao longo da história humana, el es têm sido sempre associados à imagem de poder, beleza e mobilidade social ${ }^{4}$. A sociedade atual é fortemente influenciada pela mídia, queconstrói eimpõe um padrão de beleza que valoriza a magreza e rejeita o corpo gordo ${ }^{5,6}$. Enquanto antes a gordura era vista como sinal de poder e abundância, hoje ela está associada a atributos negativos como falta de força de vontade e não conformidade com a sociedade contemporânea. Em contrapartida, o corpo magro écultuado edesejado, sendo associado à felicidade, à satisfação, à beleza, à atração sexual e à juventude ${ }^{5,7}$. Como consequência, em busca de um padrão aceito e cobrado pela sociedade, vem crescendo a insatisfação das pessoas com a própria aparência, num contexto no qual há uma grande valorização de corpos fortes e magros ${ }^{4}$.

Nesse cenário, a adolescência (definida pela Organização M undial da Saúde como a faixa entre 10 e 19 anos de idade) é um período da vida que merece particular aten ção. Caracterizada por diversas modificações físicas, comportamentais e psicossociais, entre outras ${ }^{8,9}$, ela é marcada por transformações relacionadas à formação de sua autoimagem do indivíduo. A diversidade e a intensidade dessas mudanças, aliadas a atitudes de rebeldia, à tentativa de independência, ao desejo de transgressão e à falta de preocupação com um futuro distante, podem influenciar os seus hábitos alimentares e outros comportamentos dos adolescentes quepodem repercutir sobresua saúde e bem-estar ${ }^{10-12}$. Além disso, influenciadas pelos padrões socioculturais de cada realidade, pela mídia e pela busca do corpo ideal, as alterações que acontecem no corpo, características da puberdade, assumem importância fundamental na percepção de imagem corporal que 0 adolescente tem de si mesmo, podendo gerar distorções ein satisfações da imagem corporal queacarretem atitudes relacionadas ao corpo, à alimentação e à atividade física prejudiciais a seu desenvolvimento e a sua saúde ${ }^{7,13-15}$.

A não aceitação dos adolescentes em relação a seu corpo está positivamente associada à depressão e a distúrbios alimentares como anorexia e bulimia ${ }^{16}$. Além disso, tem sido registrado aumento acelerado das prevalências de excesso de peso e de obesidade entre adolescentes em diversas partes do mundo, inclusive no Brasil ${ }^{17}$. Por esses e outros motivos (como a exposição a fatores de risco ligados ao consumo de bebidas lícitas eilícitas, violência urbana edoméstica, práticas sexuais, entreoutros), essegrupo populacional deve ser alvo de políticas públicas de promoção da saúde e do peso saudável, bem como da vigilância de comportamentos e fatores de risco e proteção à saúde.

Com vistas a subsidiar a formulação e o redirecionamento de ações e programas voltados para esse grupo, a Secretaria de Vigilância em Saúde do M inistério da Saúde, em parceria com - M inistério da Educação e com o Instituto Brasileiro de Geografia e Estatística, implementou um sistema de vigilância de fatores de risco para a saúde de adolescentes baseado em inquéritos regulares no âmbito escolar, entendendo a escola como um espaço privilegiado, não só por oportunizar a coleta dessas informações, mas também como lócus para o desenvolvimento deações de promoção da saúde junto a esse grupo etário.

0 desenho e a implementação desse sistema foram inspirados na recomendação da Organização Mundial da Saúde de implantação e manutenção de sistemas de vigilância de fatores de risco à saúde dirigidos a adolescentes, nas experiências de sistemas de outros países (Youth Risk Behavior Surveillance System - YRBSS; e H ealth Behaviour in School-aged Children - HBSC) ${ }^{18,19}$ e em experiências prévias brasileiras ${ }^{20-22}$. 0 primeiro inquérito (Pesquisa Nacional deSaúde Escolar - PeN SE) foi realizado no ano de 2009.

0 objetivo do presente trabal ho foi descrever a concordância entre imagem corporal e estado nutricional e verificar a associação de imagem corporal e de estado nutricional com atitudes e práticas relacionadas ao peso corporal entre adolescentes estudados na PeN SE 2009. 
M etodologia

População do estudo e amostragem

A população de estudo foi composta por alunos que cursavam em 2009 o $9^{\circ}$ ano do ensino fundamental de escolas públicas e privadas das 26 capitais de estados brasileiros e do Distrito Federal. O Censo Escolar 2007, realizado pelo Instituto Nacional de Estudos e Pesquisas Educacionais Anísio Teixeira, do M inistério da Educação, serviu como cadastro base, a partir do qual foi definida a amostra.

0 desenho da amostra foi estruturado de modo a representar o conjunto de estudantes do 9 o ano de escolas públicas e privadas das capitais brasileiras e do Distrito Federal. Trata-se de uma amostra complexa que envolve estratificação e conglomeração, com seleção em dois estágios. Os estratos amostrais corresponderam às capitais das unidades da federação e ao Distrito $\mathrm{Fe}-$ deral, totalizando 27 estratos. Em cada um desses estratos, as escolas que possuíam classes do 9o ano do ensino fundamental foram agrupadas em escolas privadas ou públicas. Cada estrato correspondeu a um domínio de interesse para a divulgação de resultados da pesquisa. 0 sorteio das escolas (unidade primária de amostragem) dentro de cada estrato foi feito por amostragem sistemática com probabilidade proporcional ao número de escolas nos estratos. Cada uma das escolas selecionadas nesse primeiro estágio foi visitada para a construção de uma lista atualizada de turmas do go ano do ensino fundamental existentes em 2009. 0 sorteio dasturmas (unidade secundária de amostragem), dentro de cada escola selecionada, ocorreu quando essa possuía duas ou mais turmas do 90 ano. Foi escolhida uma turma em cada escola selecionada quetivesse uma ou duas turmas do go ano do ensino fundamental, e duas turmas em cada escola com três ou mais turmas do 9o ano do ensino fundamental. Em cada uma das turmas do 90 ano do ensino fundamental selecionadas, todos os escolares responderam ao questionário da pesquisa, eliminando-se, desta maneira, a necessidade de um terceiro estágio de seleção. No período, foram estudados 63.411 estudantes. Do total de escolares presentes na data da pesquisa, nas turmas selecionadas, 501 escolares se negaram a participar da pesquisa, restando 62.910 que preencheram os questionários. Para as análises do presente artigo, utilizaram-se informações dos escolares que concordaram em participar e que responderam à variável sexo, totalizando 60.973 escolares distribuídos em 1.453 escolas e 2.175 turmas. 0 peso amostral usado nas análises de dados foi atribuído a cada um dos estudantes da amostra final, levando em conta as perdas amostrais citadas aqui. M ais informações do procedimento amostral encontram-se disponíveis em outra publicação ${ }^{23}$.

\section{Coleta de dados}

A coleta de dados foi realizada por meio de microcomputador de mão (Personal Digital Assistant - PDA), que continha questionário estruturado e autoaplicável dividido em módulos por assunto: características sociodemográficas, alimentação, imagem corporal, atividade física, tabagismo, consumo de álcool e outras drogas, saúde bucal, comportamento sexual, violência, acidentes, segurança emedidas antropométricas.

A aferição de medidas antropométricas foi realizada por profissionais treinados e padronizados segundo técnicas propostas por Lohman (1988) e Habitch (1974) ${ }^{24,25}$. O peso foi aferido em kg por balança digital portátil com capacidade máxima de 200 kg eprecisão de 0,1 kg. A altura foi medida com auxílio de estadiômetro portátil com altura máxima de $200 \mathrm{~cm}$ e precisão de $0,1 \mathrm{~cm}$.

\section{Variáveis analisadas \\ e construção de indicadores}

As variáveis referentes à imagem corporal e às atitudes em relação ao peso foram construídas com base em quatro perguntas: "Como você descreve seu peso?"; ; 0 que você está fazendo em relação ao seu peso?"; "Nos últimos 30 dias, você vomitou ou tomou laxantes para perder peso ou evitar ganhar peso?"; ; "Nosúltimos 30 dias, você tomou algum remédio, fórmula ou outro produto para perder ou manter seu peso sem acompanhamento médico?". Essas variáveis foram categorizadas conforme descrito a seguir: imagem corporal: magro (muito magro(a) + magro(a)), normal e gordo (gordo(a) + muito gordo(a)); atitude em relação ao peso: nenhuma, tenta perder peso, tenta ganhar peso e tenta manter peso. Para estudo das práticas extremas para controle ou perda do peso, foram construídas três variáveis: uso de laxante ou indução de vômito, uso de medicamento ou fórmula sem acompanhamento médico, realização de pelo menos um dos dois procedimentos anteriores.

$O$ estado nutricional (EN) foi classificado em baixo peso, adequação e excesso de peso segundo Índice de Massa Corporal - IMC (peso em 
$\mathrm{kg} /\left(\right.$ altura em $\left.\mathrm{m}^{2}\right)$ ) para idade e sexo de acordo com as distribuições preconizadas pela O M S ${ }^{26}$.

Além das variáveis demográficas sexo e idade ( $\leq 13 ; 14 ; 15 ; \geq 16$ anos) e da dependência administrativa da escola (pública e privada), foram adotados dois marcadores socioeconômicos: nível de escolaridadematerna (ensino fundamental incompleto; ensino fundamental completo; ensino médio completo; ensino superior completo) e o escore de bens e serviço (EBS) (classificado em terços da distribuição observada na amostra estudada). A construção desse escore está detal hada no artigo de Levy et al. ${ }^{27}$ "Consumo e comportamento alimentar entre adolescentes brasileiros: Pesquisa Nacional de Saúde do Escolar (PeN SE), 2009", disponível neste suplemento.

Análise dos dados

A descrição das variáveis sociodemográficas foi realizada para o total da população e por sexo do adolescente.

Para verificação da concordância entre o estado nutricional aferido pelos pesquisadores e a imagem corporal relatada pelo estudante, ambos coletados no dia da pesquisa, foi estimado 0 Kappa ponderado, arbitrando-se os seguintes pesos: peso 1 para a concordância entre as categorias de estado nutricional "magreza" eimagem corporal "magro", estado nutricional "adequado" e imagem corporal "normal" e estado nutricional "excesso de peso" eimagem corporal "gordo"; peso 0,5 para concordância entre as categorias de estado nutricional "magreza" e imagem corporal "normal", estado nutricional "adequado" e imagem corporal "magro" ou "gordo" e estado nutricional "excesso depeso" eimagem corporal "normal"; e peso 0 para as categorias extremas discordantes, ou seja, estado nutricional "magreza" e imagem corporal "gordo" e estado nutricional "excesso de peso" e imagem corporal "magro".

0 exame da eventual diferença entre os sexos tanto no que diz respeito à concordância entre estado nutricional e imagem corporal quanto às atitudes e práticas extremas em relação ao peso segundo as categorias deestado nutricional eimagem corporal foi realizado por meio de modelos de regressão logística, tomando-se como variável explanatória o sexo e como variável dependente cada uma das combinações das categorias das variáveis de interesse. Por exemplo, no caso da concordância entre estado nutricional e imagem corporal, foram examinadas as combinações imagem corporal "magro" + estado nutricional "baixo peso"; imagem corporal "magro" + estado nutricional "normal"; imagem corporal "magro" + estado nutricional "excesso de peso" e assim por diante. No caso das práticas extremas segundo estado nutricional e imagem corporal, foram examinadas as combinações imagem corporal "magro" + atitude em relação ao peso "tentou ganhar"; estado nutricional "adequado" + uso de laxante ou indução de vômito" etc.

Foram estimados dois modelos para cada uma das combinações construídas: um bivariado, somente com sexo como variável independente, e outro múltiplo, ajustando-se para as variáveis dependência administrativa da escola, idade (em anos) do aluno, EBS e escolaridade materna. Todas as análises foram realizadas respeitando a complexidade do desenho da amostra com o auxílio do aplicativo Stata 11.0 (Stata Corp College Station).

\section{Aspectos éticos}

A PeNSE-2009 foi aprovada pelo ComitêNacional deÉtica em Pesquisa do M inistério da Saúde, sob o parecer no 11.537. A participação dos alunos foi voluntária. 0 questionário poderia ser respondido em sua totalidade ou em parte. Todas as informações tanto do aluno quanto da escola foram confidenciais e não identificadas.

\section{Resultados}

Do total de alunos estudados, $72,9 \%$ estudavam em escolas públicas e cerca de metade tinha 14 anos de idade. Em relação ao EBS, maior proporção de meninos encontrava-se no terço superior e, de meninas, no terço inferior. A distribuição da amostra segundo escolaridade da mãe indicou proporções similares (de aproximadamente $1 / 3$ ) de baixa escolaridade (ensino fundamental incompleto) ealta escolaridade (superior completo) (Tabela 1).

Verificou-se baixa prevalência de baixo peso entreos adolescentes $(2,7 \%)$, sendo ligeiramente inferior entre as meninas. No entanto, em torno de $20 \%$ desses estudantes se consideraram magros ou muito magros. Já o excesso de peso atingia $24 \%$ dos alunos, sendo essa prevalência semelhanteentreos sexos. Entreas meninas, 21,3\% se consideravam gordas, enquanto $13,8 \%$ dos meninos se viam desta forma. Observou-se, ainda, que aproximadamente $2 / 3$ desses adolescentes estavam fazendo algo em relação ao seu peso, sendo esta atitude mais frequente entre as meni- 
nas. Registrou-se, também, que 7\% dos adolescentes haviam adotado, no mês que antecedeu 0 estudo, ao menos uma conduta extrema para controle ou perda de peso, sendo a frequência dessa prática bastante similar entre meninos e meninas (Tabela 1).

Para o total de alunos, foram observadas maiores proporções nas categorias de imagem corporal condizentes com cada categoria de estado nutricional, isto é: entre os alunos com baixo peso, a maior proporção se classificou como "magro"; entre os com peso adequado, a maior proporção se classificou como "normal" e, entre os com excesso de peso, a maior proporção se classificou como "gordo". Esse mesmo padrão foi observado entre meninas, mas não entremeninos, grupo no qual se observou maior proporção de autoimagem "normal" entre os com excesso de peso (52\%) (Tabela 2).

A maior proporção observada de uma categoria de imagem corporal condizente com a de estado nutricional foi aquela referente à imagem corporal "magro" entre adolescentes com baixo peso, tanto para o total de alunos $(74,9 \%)$ quan-

Tabela 1. Características sociodemográficas, estado nutricional, imagem corporal, atitude e procedimentos em relação ao peso corporal por sexo. Escolares do 9o ano nos municípios das capitais e no Distrito Federal. PenSE - 2009.

\begin{tabular}{|c|c|c|c|c|c|c|}
\hline \multirow{2}{*}{ Variáveis } & \multicolumn{2}{|c|}{ Total } & \multicolumn{2}{|c|}{ Meninos } & \multicolumn{2}{|c|}{ Meninas } \\
\hline & $\mathrm{n}$ & $(\%)$ & $\mathrm{n}$ & $(\%)$ & $\mathrm{n}$ & $(\%)$ \\
\hline \multicolumn{7}{|l|}{ Idade (anos) } \\
\hline$\leq 13$ & 15.697 & 24,5 & 6.264 & 21,1 & 9.433 & 27,5 \\
\hline 14 & 27.243 & 47,1 & 12.457 & 45,8 & 14.786 & 48,2 \\
\hline 15 & 11.141 & 18,3 & 5.844 & 20,8 & 5.297 & 15,9 \\
\hline$\geq 16$ & 6.725 & 10,2 & 3.768 & 12,3 & 2.957 & 8,3 \\
\hline \multicolumn{7}{|l|}{ Terços de escore de bens } \\
\hline 1 & 24.024 & 34,0 & 10.188 & 30,7 & 13.836 & 37,0 \\
\hline 2 & 18.127 & 32,8 & 8.564 & 33,3 & 9.563 & 32,3 \\
\hline 3 & 18.822 & 33,2 & 9.671 & 36,0 & 9.151 & 30,7 \\
\hline \multicolumn{7}{|l|}{ Escolaridade materna } \\
\hline Fundamental incompleto & 22.119 & 36,7 & 9.482 & 33,7 & 12.637 & 39,4 \\
\hline Fundamental completo & 8.100 & 13,6 & 3.746 & 13,7 & 4.354 & 13,5 \\
\hline M édio completo & 11.558 & 17,5 & 6.012 & 19,1 & 5.546 & 16,0 \\
\hline Superior completo & 19.196 & 32,2 & 9.183 & 33,5 & 10.013 & 31,1 \\
\hline \multicolumn{7}{|l|}{ Dependência administrativa } \\
\hline Privada & 14.532 & 20,8 & 7.147 & 21,3 & 7.385 & 20,4 \\
\hline Pública & 46.441 & 79,2 & 21.276 & 78,7 & 25.165 & 79,6 \\
\hline \multicolumn{7}{|l|}{ Estado nutricional } \\
\hline Baixo peso & 1.626 & 2,7 & 948 & 3,4 & 678 & 2,0 \\
\hline Adequação & 45.327 & 73,3 & 20.599 & 71,7 & 24.728 & 74,9 \\
\hline Excesso de peso & 13.853 & 24,0 & 6.786 & 25,0 & 7.067 & 23,1 \\
\hline \multicolumn{7}{|l|}{ Imagem corporal: } \\
\hline Magro & 13.180 & 22,1 & 6.288 & 23,0 & 6.892 & 21,4 \\
\hline Normal & 35.663 & 60,2 & 17.412 & 63,3 & 18.251 & 57,4 \\
\hline Gordo & 10.618 & 17,7 & 3.805 & 13,8 & 6.813 & 21,3 \\
\hline \multicolumn{7}{|l|}{ Atitude em relação ao peso: } \\
\hline Nenhuma & 21.649 & 37,2 & 10.731 & 39,8 & 10.981 & 35,0 \\
\hline Tentava perder & 16.184 & 27,5 & 5.636 & 20,9 & 10.548 & 33,3 \\
\hline Tentava ganhar & 9.687 & 15,1 & 5.405 & 17,9 & 4.282 & 12,6 \\
\hline Tentava manter & 11.933 & 20,2 & 5.755 & 21,4 & 6.178 & 19,1 \\
\hline \multicolumn{7}{|c|}{ Práticas extremas para perder ou manter o peso } \\
\hline U so de laxante ou indução de vômito & 2.407 & 4,2 & 890 & 3,5 & 1.517 & 4,8 \\
\hline U so de medicamento ou fórmula & 2.387 & 4,2 & 1.243 & 4,9 & 1.144 & 3,6 \\
\hline $\begin{array}{l}\text { U so de pelo menos um dos dois } \\
\text { procedimentos }\end{array}$ & 3.974 & 6,9 & 1.761 & 6,6 & 2.213 & 7,0 \\
\hline
\end{tabular}


to para cada um dos sexos (meninos: $71,1 \%$; e meninas: $80,7 \%$ ). Já a maior proporção observada entre uma categoria de imagem corporal discordante da categoria de estado nutricional foi aquela referente à imagem corporal "normal" entreadolescentescom excesso depeso (total: $47,2 \%$; meninos: 52,0\%; meninas: 42,6\%) (Tabela 2).

Foram observadas diferenças estatisticamente significativas entre meninos e meninas para quase todas as proporções de combi nações entre imagem corporal e estado nutricional, diferenças essas que se mantiveram mesmo após ajuste para variáveis sociodemográficas. As proporções de combinações que apresentaram maior contraste relativo (razão de prevalências [RP] supe rior a 1,5) entre os sexos foram as seguintes: indivíduos com baixo peso que se viam gordos (meninos: 1,6\%; meninas: 0,9\%; RP=1,8); indivíduos com peso adequado que se viam gordos (meninas: $11,3 \%$; meninos: 3,3\%; RP=3,4); e indivíduos com excesso de peso que se viam magros (meninos: 2,9\%; meninas: 1,5\%; RP=1,9) (Tabela 2).

Entretanto, em conjunto, foi baixa a concordância entre o estado nutricional do estudante e sua imagem corporal no momento da pesquisa, com valores estimados de Kappa ponderado de $0,33,0,34$ e 0,32 para o total de alunos, meninos emeninas, respectivamente.

Do total de adolescentes, $54 \%$ dos que apresentavam baixo peso estavam tentando ganhar peso, $77,2 \%$ dos que tinham excesso de peso estavam tentando perder ou manter o peso e $57,7 \%$ dos queestavam com estado nutricional adequado tentavam ganhar, perder ou manter o peso (Tabela 3). O bservou-se, também, que 21,8\% daqueles com excesso de peso não estavam ten- do nenhuma atitude em relação ao peso. Foram registradas, ainda, diferenças estatisticamentesignificativas entre meninos emeninas no tocante à atitudeem relação ao peso entreaqueles com peso adequado e excesso de peso. Por exemplo, comparadas aos meninos, maior proporção de me ninas tentava perder peso quando elas apresentavam estado nutricional adequado ou excesso de peso. Em comparação com as meninas, maior proporção de meninos tentava ganhar peso quando apresentava estado nutricional adequado e não tomava nenhuma atitude quando apresentava excesso de peso (Tabela 3).

Quando examinadas as atitudes dos adolescentes em relação ao seu peso segundo sua imagem corporal, observou-se que $43,6 \%$ dos quese consideravam magros estavam tentando ganhar peso, $79,2 \%$ dos que se consideravam gordos estavam tentando perder ou manter o peso e 59,1\% dos que se consideravam com peso normal tentavam ganhar, perder ou manter o peso (Tabela 3). Observou-se, também, que $20,2 \%$ daqueles que se consideravam gordos não estavam tendo nenhuma atitude em relação ao peso, não havendo diferença entre os sexos. Foram registradas, ainda, diferenças estatisticamente significativas entremeninos emeninas no tocanteà atitude em relação ao peso em praticamente todas as categorias deimagem corporal examinadas. Cabe destacar, aqui, a diferença de comportamento entre meninos e meninas quando se consideravam com peso normal: maior proporção de meninas tentava perder peso, enquanto maior proporção de meninos tentava ganhar peso (Tabela 3). Outra diferença importante entre os sexosfoi observada quando os adolescentes se consideravam magros: maior proporção de meni-

Tabela 2. Imagem corporal segundo estado nutricional por sexo. Escolares do 9o ano nos municípios das capitais e no Distrito Federal. PenSE - 2009.

\begin{tabular}{|c|c|c|c|c|c|c|c|c|c|c|c|c|}
\hline \multirow{3}{*}{$\begin{array}{l}\text { I magem } \\
\text { corporal }\end{array}$} & \multicolumn{12}{|c|}{ Estado nutricional } \\
\hline & \multicolumn{3}{|c|}{ Meninos } & \multicolumn{4}{|c|}{ Meninas } & \multirow[b]{2}{*}{ k } & \multicolumn{3}{|c|}{ Total } & \multirow[b]{2}{*}{$\mathrm{k}$} \\
\hline & $\begin{array}{l}\text { Baixo } \\
\text { peso }\end{array}$ & Adequação & $\begin{array}{l}\text { Excesso } \\
\text { de peso }\end{array}$ & $\mathrm{k}$ & $\begin{array}{l}\text { Baixo } \\
\text { peso }\end{array}$ & Adequação & $\begin{array}{l}\text { Excesso } \\
\text { de peso }\end{array}$ & & $\begin{array}{l}\text { Baixo } \\
\text { peso }\end{array}$ & Adequação & $\begin{array}{l}\text { Excesso } \\
\text { de peso }\end{array}$ & \\
\hline M agro & $71,1^{a, b^{*}}$ & $27,8^{a, b}$ & $2,9^{a, b}$ & 0,34 & 80,7 & 25,9 & 1,5 & 0,32 & 74,9 & 26,7 & 2,2 & 0,33 \\
\hline Normal & $27,4^{a, b *}$ & $68,9^{a, b}$ & $52,0^{a, b}$ & & 18,5 & 62,9 & 42,6 & & 23,8 & 65,7 & 47,2 & \\
\hline Gordo & $1,6^{*}$ & $3,3^{a, b}$ & $45,2^{a, b}$ & & 0,9 & 11,3 & 55,9 & & 1,3 & 7,6 & 50,6 & \\
\hline
\end{tabular}

*M odelo sem ponderação devido à existência de estrato com apenas uma unidadeamostral; ; ${ }^{2}$ Diferença estatisticamente significativa $(p<0,05)$ entre os sexos em modelo bruto entre sexo e desfechos de interesse; ${ }^{b}$ Diferença estatisticamente significativa $(p<0,05)$ entre os sexos em modelo ajustado para terços deescore de bens, escolaridade materna, dependência administrativa da escola eidade do aluno. 
nos tentava ganhar peso, enquanto maior proporção de meninas tentava mantê-lo.

Haviam recorrido à adoção de pelo menos uma prática extrema para manter ou perder peso, no mês que antecedeu o estudo, 9,4\% dos adolescentes com excesso de peso e 11,3\% dos quese consideravam gordos; $6,1 \%$ daqueles com estado nutricional adequado e 5,5\% dos que se consideravam com peso normal; e 5,8\% daqueles com baixo peso e $6,9 \%$ dos que se consideravam magros. Diferenças estatisticamente significativas entre os sexos foram observadas entre aqueles com peso adequado e excesso de peso e prati- camente para todas as categorias de imagem corporal. Entreos adolescentes com excesso de peso e entre aqueles que se consideravam obesos, mai or proporção de meninas recorria a práticas extremas para controle do peso (Tabela 3 ).

\section{Discussão}

Os resultados aqui apresentados indicaram, entre os adolescentes das capitais brasileiras de ambos os sexos, ser relevante a prevalência de excesso de peso ( $24 \%)$, e que $27,5 \%$ dos jovens

Tabela 3. Comportamentos em relação ao peso segundo estado nutricional e imagem corporal por sexo. Escolares do 9o ano nos municípios das capitais e no Distrito Federal. PenSE - 2009.

\begin{tabular}{|c|c|c|c|c|c|c|}
\hline \multirow[t]{2}{*}{ Variáveis } & \multicolumn{3}{|c|}{ Estado nutricional } & \multicolumn{3}{|c|}{ Imagem corporal } \\
\hline & $\begin{array}{l}\text { Baixo } \\
\text { peso }\end{array}$ & A dequação & $\begin{array}{l}\text { Excesso } \\
\text { de peso }\end{array}$ & Magro & Normal & Gordo \\
\hline \multicolumn{7}{|l|}{ Meninos } \\
\hline \multicolumn{7}{|l|}{ Atitude em relação ao peso } \\
\hline Nenhuma & $38,2 *$ & $45,2^{a, b}$ & $24,5^{\mathrm{a}, \mathrm{b}}$ & 40,8 & $43,6^{a, b}$ & 20,5 \\
\hline Tentava perder & $3,2^{*}$ & $8,6^{a, b}$ & $58,6^{a, b}$ & $2,6^{a, b}$ & $16,0^{a, b}$ & $74,0^{b}$ \\
\hline Tentava ganhar & $50,1^{*}$ & $22,2^{a, b}$ & $1,5^{\mathrm{a}, \mathrm{b}}$ & $47,3^{a, b}$ & $11,0^{a, b}$ & 0,8 \\
\hline Tentava manter & $8,5^{*}$ & $24,1^{b}$ & $15,4^{a, b}$ & $9,3^{\mathrm{a}, \mathrm{b}}$ & $29,4^{a, b}$ & $4,8^{a, b}$ \\
\hline \multicolumn{7}{|l|}{ Práticas extremas para perder ou manter o peso*: } \\
\hline U so de laxante ou indução de vômito & $1,8^{*}$ & $3,4^{b}$ & $4,0^{a, b}$ & $4,4^{a, b}$ & $3,0^{b}$ & $4,1^{a, b}$ \\
\hline Uso de medicamento ou fórmula & $4,5^{*}$ & $4,8^{a, b}$ & 5,2 & $7,5^{\mathrm{a}, \mathrm{b}}$ & $4,0^{a, b}$ & $4,7^{a, b}$ \\
\hline U so de pelo menos um dos dois procedimentos & $5,8^{*}$ & 6,5 & $7,2^{a, b}$ & $8,7^{\mathrm{a}, \mathrm{b}}$ & 5,7 & $7,2^{a, b}$ \\
\hline \multicolumn{7}{|l|}{ Meninas } \\
\hline \multicolumn{7}{|l|}{ Atitude em relação ao peso } \\
\hline Nenhuma & 33,3 & 39,9 & 19,2 & 40,8 & 38,3 & 20,1 \\
\hline Tentava perder & 2,4 & 22,6 & 71,0 & 4,9 & 27,9 & 76,2 \\
\hline Tentava ganhar & 59,9 & 15,0 & 0,5 & 40,1 & 6,9 & 0,4 \\
\hline Tentava manter & 4,4 & 22,6 & 9,3 & 14,3 & 26,9 & 3,3 \\
\hline \multicolumn{7}{|l|}{ Práticas extremas para perder ou manter o peso*: } \\
\hline U so de laxante ou indução de vômito & 1,1 & 3,8 & 8,2 & 2,8 & 3,6 & 9,8 \\
\hline Uso de medicamento ou fórmula & 5,1 & 2,9 & 5,7 & 3,2 & 2,6 & 6,6 \\
\hline U so de pelo menos um dos dois procedimentos & 5,7 & 5,7 & 11,5 & 5,2 & 5,3 & 13,6 \\
\hline \multicolumn{7}{|l|}{ Total } \\
\hline \multicolumn{7}{|l|}{ Atitude em relação ao peso } \\
\hline Nenhuma & 36,3 & 42,3 & 21,8 & 40,8 & 40,9 & 20,2 \\
\hline Tentava perder & 2,9 & 16,1 & 64,9 & 3,8 & 22,0 & 75,4 \\
\hline Tentava ganhar & 54,0 & 18,3 & 1,0 & 43,6 & 9,0 & 0,5 \\
\hline Tentava manter & 6,8 & 23,3 & 12,3 & 11,8 & 28,1 & 3,8 \\
\hline \multicolumn{7}{|l|}{ Práticas extremas para perder ou manter o peso*: } \\
\hline U so de laxante ou indução de vômito & 1,5 & 3,6 & 6,1 & 3,6 & 3,3 & 7,7 \\
\hline Uso de medicamento ou fórmula & 4,7 & 3,8 & 5,4 & 5,3 & 3,3 & 5,9 \\
\hline U so de pelo menos um dos dois procedimentos & 5,8 & 6,1 & 9,4 & 6,9 & 5,5 & 11,3 \\
\hline
\end{tabular}

*M odelo sem ponderação devido à existência de estrato com apenas uma unidade amostral; ** Sem acompanhamento médico; ${ }^{\text {a }}$ Diferença estatisticamente significativa $(p<0,05)$ entre os sexos em modelo bruto entre sexo e desfechos de interesse; ${ }^{b}$ Diferença estatisticamente significativa $(p<0,05)$ entre os sexos em modelo ajustado para terços deescore de bens, escolaridade materna, dependência administrativa da escola eidade do aluno. 
tentavam perder ou manter o peso, já sendo re corrente $o$ uso de práticas extremas para controle do peso (6,9\%). Revelaram, ainda, as percepções de imagem corporal segundo estado nutricional dos adolescentes.

A comparação de nossos resultados com os deoutros sistemas demonitoramento não ésimples, tendo em vista as diferenças observadas na faixa etária dos adol escentes estudados ena construção dos indicadores examinados. Em que pe sem essas diferenças, é possível uma comparação de alguns dos achados descritos neste estudo com os de dois sistemas de vigilância de fatores de risco dirigidos a adolescentes: o europeu $(\text { HBSC })^{16}$ e 0 americano (YRBSS) ${ }^{28}$.

Em relação ao estado nutricional, o HBSC2005/2006 ${ }^{16}$ apontou prevalência de $13 \%$ de excesso de peso (entreadolescentes com 15 anos de idade). Já o YRBSS-2009 identificou prevalência de $27,8 \%{ }^{27}$. Os resultados do estudo brasileiro (24\%) apresentam uma situação intermediária, mais próxima da observada entre os adolescentes dos Estados Unidos.

Com relação a comportamentos para controle do peso, no HBSC-2005/2006 o indicador utilizado foi a proporção de adolescentes que informaram "fazer dieta ou alguma outra coisa para perder peso". Entre os adolescentes com 15 anos de idade, $15 \%$ relataram ter essa prática ( $23 \%$ das meninas contra $8 \%$ dos meninos) ${ }^{16}$. Já no YRBSS-2009, o indicador utilizado foi a proporção de adolescentes que informaram "estar tentando perder peso" 7 . Entre os adolescentes estudados, $44,4 \%$ relataram essa prática ( $59,3 \%$ entre meninas; $30,5 \%$ entre meninos). Os resultados brasileiros (27,5\% relataram tentar perder peso; meninos: $20,9 \%$; meninas: $33 \%$ ), mais uma vez, ficam numa situação intermediária entre os resultados europeus e os americanos em termos de frequência do comportamento examinado e se mostram similares em relação ao fato de essa prática ser mais recorrente entre meninas.

No tocantea práticas extremas para controle do peso, é possível comparar nosso estudo somente com o YRBSS-2009. Os indicadores gerados por esse sistema são a proporção de adolescentes que relataram praticar, nos trinta dias que antecederam o estudo, os seguintes comportamentos: uso de laxante ou vômito para manter ou perder peso euso de medicamentos sem aconselhamento médico para perder ou manter peso ${ }^{28}$. Do total de adolescentes, $4 \%$ (meninas: 5,4\%; meninos: $2,6 \%$ ) relataram ter realizado a primeira prática e $5 \%$ (meninas: $6,3 \%$; meninos: 3,8\%), a segunda prática. Esses resultados são semel han- tes aos observados em nosso estudo, em que se registrou que $4,2 \%$ dos jovens (meninas: $4,8 \%$; meninos: $3,5 \%$ ) relataram realizar a primeira prática e 4,2\% (meninas: 3,6\%; meninos: 4,9\%), a segunda.

Ainda sobre as práticas extremas para controle do peso aqui examinadas, cabe dizer que, enquanto não existem dados sobre a magnitude de transtornos alimentares como bulimia e anorexia nervosa entreadolescentes em âmbito nacional, os indicadores aqui apresentados podem ser utilizados como proxy de vulnerabilidade para (ou da presença de) esses agravos. Cabe ainda dizer que as práticas extremas estudadas na PeN SE-2009 podem indicar, na verdade, a ponta do iceberg de comportamentos não saudáveis para controledo peso, como, por exemplo, jejum prolongado, supressão de refeições, uso de dietas altamente restritivas ou com combinações inadequadas de alimentos. Ou seja, a magnitude de práticas não saudáveis para controle do peso é, no mínimo, a registrada aqui.

A concordância entreo estado nutricional do adolescente e a sua percepção de imagem corporal foi baixa neste estudo. Quando comparados os resultados entre meninos e meninas, observase que maior proporção delas identifica situações extremas (baixo peso e excesso de peso), enquanto maior proporção deles identifica a situação de adequação do estado nutricional. Parte dessa baixa concordância pode ser explicada pelo fato de este grupo etário estar em um período marcado por tran sformações biopsicossociais e desenvolvimento de sua identidade, sendo suscetível às pressões socioculturais que enfatizam, particularmente por meio da mídia, o ideal de forma física, caracterizado por uma magreza irreal para as mulheres eum corpo musculoso para os homens $5^{5,14,15}$.

Ainda em relação às percepções de imagem corporal segundo estado nutricional dos adolescentes, cabe aqui refletir sobre o propósito dessa abordagem e suas limitações, tendo em vista o grupo etário estudado e os dados disponíveis. Tradicional mente, esse tipo de análise érealizado em estudos com adultos com o intuito de se investigar o gap entre real idade e percepção (cenário objetivo e dimensão subjetiva), assumindose que três situações são indesejáveis em termos de promoção da saúde e prevenção de doenças: o indivíduo se considerar são quando apresenta algum agravo; 0 indivíduo considerar que tem algum agravo quando está são; e o indivíduo considerar que tem um agravo quando, na verdade, padece de outro. Esse tipo de abordagem 
assume o diagnóstico de saúde (no caso, a classificação do estado nutricional) como "padrãoouro" e a imagem corporal como o evento passível de distorção. Em se tratando de adolescentes, essa assunção deve ser relativizada. As classificações nutricionais disponíveis (entreel as a utilizada neste estudo) são limitadas para o âmbito individual, tendo em vista quenão levam em conta a maturação sexual do adolescente, fenômeno que apresenta grande variabilidade entre indivíduos, particularmente atéa faixa etária estudada aqui, einfluencia enormemente nas dimensões e proporções corporais. Além disso, muitas vezes os pontos de corte adotados para diagnóstico de baixo peso eexcesso de peso apresentam sensibilidade e especificidade distintas, acarretando diferentes proporções de falsos positivos e falsos negativos para o diagnóstico de agravo.

Outro aspecto a ser comentado se refere à variável construída para captar a imagem corporal do adolescente com base na pergunta "em relação ao seu peso, como vocêse vê?" e nas respostas "muito magro", "magro", "normal", "gordo" e "muito gordo". Cabe perguntar: em que medida se poderia esperar uma superposição plena entre essas categorias e as faixas de estado nutricional adotadas? 0 aluno, ao preencher 0 questionário, quando se depara com a opção "magro", pensa em uma situação de déficit pon- deral ou em uma variação da normalidade? Por outro lado, ao se deparar com a opção "gordo", imagina quesituações de excesso de peso quenão secaracterizem como obesidade clinicamenteinquestionável devam estar aí incluídas?

Em resumo, parte da discordância entre estado nutricional e imagem corporal encontrada nesteestudo pode ser explicada por limitações nos instrumentos e procedimentos de comparação utilizados. Testes de validação de instrumentos e procedimentos analíticos alternativos devem ser desenvolvidos para que essa comparação possa ser mais bem analisada neste grupo etário.

Por fim, cabedizer que, pela primeira vez, em nosso país, são disponibilizadas e examinadas de forma combinada informações de abrangência nacional sobre o trinômio imagem corporal/ estado nutricional/comportamentos para controle do peso referentes a adolescentes. Esses resultados evidenciam a importância de análises desse tipo para melhor compreensão da realidade do adolescentebrasileiro. Entretanto, na perspectiva de se subsidiarem políticas públicas, muito ainda se precisa conhecer sobre essa temática, sendo necessário, para isso, combinar pesquisas epidemiológicas com outras de abordagem qualitativa, de forma a se conhecer com mais profundidade esse complexo trinômio, que agrega componentes objetivos e subjetivos.

\section{Colaboradores}

IRR Castro, RB Levy, LO Cardoso, MD Passos, LMV Sardinha, LF Tavares, SP Dutra e A M artins participaram, igualmente, de todas as etapas de elaboração do artigo. 
1. Schilder P. A imagem do corpo: as energias construtivas da psique. São Paulo: Martins Fontes; 1981.

2. Ricciardelli LA, McCabe MP, Banfield S. Sociocultural influences on body image and body changes methods. J Adolesc Health 2000; 26(1):3-4.

3. Cash TF, Pruzinsky T, editors. Body image: a handbook of theory, research, and clinical practice. New York: Guilford; 2002.

4. Penna L. Imagem corporal: uma revisão seletiva da literatura. Psicol USP 1990; 1(2):167-174.

5. Pereira EF, Graup S, Lopes AS, Borgatto AF, Daronco LSE. Percepção da imagem corporal de crianças e adolescentes com diferentes níveis socioeconômicos na cidade de Florianópolis, Santa Catarina, Brasil. Rev Bras Saúde M ater Infant 2009; 9(3):253-262.

6. Novaes JV. 0 intolerável peso da feiúra: sobre mulhe res e seus corpos. Rio de Janeiro: PUC-Rio; 2006.

7. Farias RCP. Nos bastidores da moda: um estudo sobre representações de vestuário e de imagem corporal por um grupo de pré-adolescentes [dissertação]. Viçosa, M inas Gerais: Universidade Federal de Viçosa; 2004.

8. Contreras J. La obesidad: una perspectiva sociocultural. Form Contin Nutr O bes 2002; 5(6):275-286.

9. World Health Organization. Physical Status: the use and interpretation of anthropometry. Geneva: WHO; 1995.

10. Kazapi IM, Di Pietro PF, Avancini SRP, Freitas SFT, Tramonte VLCG. Consumo de energia e macronutrientes por adolescentes de escolas públicas e privadas. Rev Nutr 2001; 14(Supl):27-33.

11. Lemos M CM, Dallacosta MC. Hábitos alimentares de adolescentes: conceitos e práticas. Arq Ciênc Saúde Unipar 2005; 9(1):3-9.

12. Branco LM, Hilário MOEC, Pádua I. Percepção e satisfação corporal em adolescentes e a relação com seu estado nutricional. Rev Psiq Clín 2006; 33(6):292296.

13. Pacheco SSM . O hábito alimentar enquanto um comportamento culturalmente produzido. In: Freitas MCS, Fontes GAV, Oliveira N, organizadores. Escritos e narrativas sobre alimentação e cultura. Salvador: EdUFBA; 2008.

14. Nunes MA, Olinto MTA, Barros F, Camey S. Influência da percepção do peso e do índice de massa corporal nos comportamentos alimentares anormais. Rev Bras Psiquiatr 2001; 23(1):21-27.

15. Conti MA, Frutuoso M FP, Gambardella AMD. Excesso de peso e insatisfação corporal em adolescentes. Rev Nutr 2005; 18(4):491-497.

16. Neumark-Sztainer D, Hannan PJ, Story M, Croll J, Perry C. Family meal patterns: associations with sociodemographic characteristics and improved dietary intake among adolescents. J Am Diet Assoc 2003; 103(3):317-322.

17. World Health Organization. Inequalities in young people's health: Health Behaviour in School-aged Children (HBSC): international report from the 2005/2006 survey $\mathrm{H}$ ealth Policy for Children and Adolescents $n$. 5. Geneva: WHO Europe; 2008.

18. Conde WL, M onteiro CA. Secular changes of overweight among Brazilian adolescents from 1974/5 to 2002/3. In: O'Dea JA, Ericksen M, editors. Childhood obesity prevention. Oxford: Oxford University Press; 2010.
19. Brener ND, Kann L, Kinchen SA, Grunbaum JA, Whalen L, Eaton D, Hawkins J, Ross JG. Methodology of the youth risk behavior surveillance system. M M WR Recomm Rep 2004; 53:1-13.

20. Currie $C$, Roberts $C$, M organ A, Smith R, Settertobulte W, Samdal O, Barnekox Rasmussen V. Young people's health in context: $\mathrm{H}$ ealth Behaviour in Schoolaged Children (H BSC) study: international report from the 2001/2002 survey. Geneva: World Health Organization; 2004. (Health Policy for Children and Adolescents, 4).

21. Castro IRR, Cardoso LO, Egstrom EM, Levy RB, M onteiro CA. Vigilância de fatores de risco para doenças não transmissíveis entre adolescentes: a experiência da cidade do Rio de Janeiro, Brasil. Cad Saude Publica 2008; 24(10):2279-2288.

22. Monteiro CA, M oura EC, Jaime PC, Lucca A, Florindo $A A$, Figueiredo ICR, Bernal R, Silva NN. Monitoramento de fatores de risco para doenças crônicas por entrevistas telefônicas. Rev Saude Publica 2005; 39(1):47-57.

23. Brasil. Ministério da Saúde. Instituto Nacional de Câncer. Vigiescola: vigilância de tabagismo em escolares: dados e fatos de 12 capitais brasileiras. v. 1. Rio Janeiro: M inistério da Saúde, Instituto Nacional de Câncer; 2004

24. Instituto Brasileiro de Geografia e Estatística. Pesquisa Nacional de Saúde Escolar. Rio de Janeiro: IBGE; 2009.

25. Lohman TG, Roche AF, Martorell R. Anthropometric standardization reference manual. Champaign, Illinois: Human Kinetics Books; 1988.

26. Habitch JP. Estandarizacion de métodos epidemiológicos cuantitativos sobre el terreno. Bol Oficina Sanit Panam 1974; 76(5):375-384.

27. Levy RB, Castro IRR, Cardoso LO, Tavares LF, Sardinha LMV, Gomes FS, Costa AWN. Consumo e comportamento alimentar entre adolescentes brasileiros. Pesquisa Nacional de Saúde do Escolar (PeN SE), 2009. Cien Saude Colet 2010; 15(Supl.2):3085-3097.

28. De Onis M, Onyango AW, Borghi E, Siyam A, Nishida C, Siekmann J. Development of a WHO growth reference for school-aged children and adolescents. Bull World Health Organ 2007; 20(85):660-667.

Artigo apresentado em 08/06/2010

Aprovado em 15/07/2010

Versão final apresentada em 05/08/2010 